\title{
Advancing the pharmacological treatment of bipolar depression
}

\author{
Sophia Frangou
}

\begin{abstract}
Bipolar disorder is a recurring, often chronic, illness characterised by periods of mania and depression with variable inter-episode recovery. For the majority of patients it is the depressive component of this illness that contributes to most of the associated morbidity, social disability and mortality. Research and clinical experience suggest that acute treatment and prevention of depressive episodes is by far the most challenging aspect of the care of patients with the disorder. This review examines the contribution of depression to the course and outcome of bipolar disorder as well as diagnostic difficulties that often complicate treatment and may lead to inappropriate medication. Key studies that form the evidence base of treatment recommendation for bipolar depression are presented and areas of therapeutic uncertainty are highlighted.
\end{abstract}

DSM-IV bipolar disorder ${ }^{1}$ is characterised by repeated episodes of mania and depression (American Psychiatric Association, 1994). DSM-IV type I bipolar disorder (bipolar I disorder) presents with episodes of mania, whereas hypomanic episodes define type II bipolar disorder (bipolar II disorder). Research and clinical experience suggest that it is the depressive component of bipolar disorder that is the most disabling and the most difficult to treat. On average, depressive episodes last longer than manic ones, with about $20 \%$ running a particularly chronic course (Keller, 1988). Despite the high prevalence and morbidity of bipolar depression, few studies have focused specifically on the depressive phase of bipolar disorder. However, the situation is changing, with new studies adding to our evidence base for the pharmacological treatment of bipolar depression.

Apart from mood stabilisers, a large number of other psychotropic drugs are used in the acute and long-term treatment of bipolar depression. Various guidelines have been developed to direct clinicians through the maze of prescribing options and algorithms (e.g. American Psychiatric Association, 2002; Goodwin et al, 2003). Those produced by the British Association for Psychopharmacology (Goodwin, 2003) may be particularly relevant for UK psychiatrists, as they have taken into account

1. The ICD-10 classification of bipolar affective disorder follows a similar subcategorisation based in part on manic or hypomanic episodes (World Health Organization, 1992). national clinical practices and service organisation in addition to providing up-to-date evidence-based recommendations. An outline of recommendations pertinent to the treatment of bipolar depression is presented in Table 1. The purpose of my review is to summarise key studies that form the evidence base of good clinical practice in the pharmacological treatment of bipolar depression and to highlight areas of therapeutic uncertainty. Psychological treatments are also available and the evidence base for their efficacy in bipolar disorder is expanding to the point that they deserve to be reviewed on their own merit.

\section{The prevalence of depression in bipolar disorder}

Although hypomanic/manic episodes define bipolar disorder, depressive symptoms tend to dominate the clinical picture and may contribute to the psychosocial disability associated with the disorder. In the National Institute of Mental Health Collaborative Depression Study (Judd et al, 2002) weekly symptomatic ratings were obtained from 146 patients with bipolar I disorder over a mean period of 12.8 years. Patients were symptomatic for $47.3 \%$ of the followup time; depressive symptoms (present for $31.9 \%$ of the follow-up weeks) predominated over manic/ hypomanic (8.9\%) and cycling/mixed states (5.9\%). Minor or subsyndromal depressive symptoms were far more common (14\% and $9 \%$ respectively) than

Sophia Frangou is reader in psychiatry and Head of the Section of Neurobiology of Psychosis at the Institute of Psychiatry (PO Box 66, De Crespigny Park, London SE5 8AF, UK. Tel/fax: 0207848 0903; e-mail: s.frangou@iop.kcl.ac.uk). Her research focuses on the pathophysiology and treatment of psychoses. Dr Frangou has received financial support from Sanofi-Synthelabo, GlaxoSmithKline and Bristol-Myers Squibb for conference attendance and consultancies and from AstraZeneca and Novartis for research. 
Table 1 Summary of recommendations based on the guidelines of the British Association for Psychopharmacology (after Goodwin et al, 2003)

Illness phase

Acute depression

For patients not already on

long-term treatment for

bipolar disorder

For patients who have a depressive episode while on long-term treatment

Long-term treatment Monotherapy options

If monotherapy fails
Recommendation

Level of

evidence ${ }^{1}$

Antidepressant monotherapy is not recommended

B

Consider treatment with an antidepressant (e.g. SSRI) together with

with an antimanic agent (e.g. lithium, valproate or an antipsychotic)

For mild to moderate depression consider initial treatment with

lamotrigine, lithium or, possibly, valproate

Consider antipsychotics for patients with psychotic symptoms

Optimise dosing of existing medication and ensure that serum

levels of lithium are within the therapeutic range

Consider adding, changing or augmenting antidepressant

medication if depression is severe and optimisation has failed

Consider lithium as initial monotherapy

Carbamazepine is less effective than lithium; it may benefit patients

who are intolerant of/poor responders to lithium and who do not

show the classic pattern of episodic euphoric mania

Valproate may be more effective against depressive than against manic recurrences

Olanzapine may be more effective against manic than against depressive recurrences

Lamotrigine is more effective against depressive than against manic recurrences

In individual patients, good clinical response to any of the above agents may be considered evidence in favour of its long-term use as monotherapy

Where mania predominates, consider combining mainly antimanic agents (e.g. lithium, valproate, an antipsychotic)

Where depression predominates, consider lamotrigine or an antidepressant in combination with an antimanic long-term agent

Consider clozapine in treatment-resistant patients
A

B

B

A

B

A

B

B

B

B

D

D

D

C

1. Level of evidence: A, from large representative population samples; B, from small, well-designed studies, or extrapolated from large representative population samples; C, from non-representative surveys or case reports, or extrapolated from small, well-designed studies; D, from expert-committee reports, opinions or clinical experience of respected authorities, or extrapolated from any of the previous levels of evidence.

major depressive episodes (9\%). In a subsequent study, Judd and colleagues focused on bipolar II disorder and obtained weekly symptomatic ratings from 86 patients over a mean period of 13.4 years (Judd et al, 2003). These patients were symptomatic for $53.9 \%$ of the time; depressive symptoms predominated, being present for $50.3 \%$ of follow-up weeks, whereas manic/hypomanic and cycling/ mixed states were present for $1.3 \%$ and $2.3 \%$ of the time respectively. As in bipolar I disorder, minor and subsyndromal depression were three times more common than major depressive episodes.

\section{Diagnostic issues}

Current diagnostic criteria do not differentiate between depressive episodes arising in the context of major depressive disorder as opposed to bipolar disorder. In addition, a diagnosis of bipolar disorder can only be made after at least one manic or hypomanic episode. Consequently, many patients are misdiagnosed and treated as having major depression either because they present with depression at illness onset or because they underreport previous manic or, more commonly, hypomanic episodes. The situation is not helped by the current lack of clear definition for hypomania, which is crucial for the diagnosis and treatment of bipolar II disorder. Angst et al (2003) argue that the DSM-IV criteria for hypomania are both restrictive and epidemiologically invalid. On the basis of an ongoing study that has examined mood symptoms longitudinally since 1978 in a representative sample of 591 individuals from the canton of Zurich, they propose that either euphoria/irritability or overactivity lasting at least 2 days and at least three out of the seven symptoms of DSM-IV hypomania 
should be sufficient for the diagnosis. This wider definition raises the prevalence rates for bipolar disorder to up to $6 \%$ in the general population and possibly up to $50 \%$ in patients with major unipolar depression (Angst et al, 2003).

Several studies have found that depressive presentations are equally or perhaps even more common than manic presentations (Lish et al, 1994; Raymont et al, 2003). As shown by the National Institute of Mental Health's Clinical Collaborative Depression Study (Akiskal, 1994), careful diagnostic assessment is not particularly helpful for those presenting with depressive onset. In this study, 559 patients were diagnosed as having major depression on the basis of structured diagnostic assessments. However, during the subsequent 11-year follow-up period, 3.9\% developed bipolar I disorder and 8.6\% bipolar II disorder. The rate of misdiagnosis in more chronic patients, owing to failure in identifying the presence of manic/hypomanic episodes, is even higher. Careful evaluation of patients with a diagnosis of major depressive disorder has led to the reclassification of between $40 \%$ and $55 \%$ as having bipolar I or bipolar II disorder (Hantouche et al, 1998; Ghaemi et al, 1999).

There are no specific symptoms or other characteristics that can reliably differentiate between unipolar and bipolar depressive episodes. However, some clinical features, such as those listed in Box 1, are suggestive of bipolar rather than unipolar disorder. Despite the considerable overlap, several studies have highlighted symptomatic differences between bipolar and unipolar depression that could sensitise clinicians to the possibility that they are dealing with a patient who has bipolar disorder. Atypical features are considered more common in bipolar disorder depression (Akiskal \& Benazzi, 2004). These include reactive mood, marked anergia, reverse vegetative symptoms and increased sensitivity to criticism. Several studies have suggested that sleep was less disturbed in bipolar disorder depression, particularly in terms of less wakefulness and early morning awakening, but rapid eye movement (REM) sleep was more fragmented (Yatham et al, 1997).

Box 1 Features suggestive of bipolar depression

- Family history of any bipolar disorder in firstdegree relatives

- Early age at onset

- High frequency of depressive episodes

- Psychotic features

- Hypersomnia and psychomotor retardation

- A period of increased energy and activity immediately preceding the depression
In one of the most recent and largest studies, Mitchell et al (2001) assessed 270 patients presenting with a major depressive episode and identified 39 with a diagnosis of bipolar I disorder. These were then matched to 39 patients with major depressive disorder and compared on symptom profile, physical health, and previous psychiatric and family history in order to identify any features that were distinctive of bipolar depression. No differences were found between the two groups in severity of the index depressive episode. Patients with bipolar disorder, however, were more likely to have psychotic symptoms during the index or previous depressive episodes and more likely to have melancholic and atypical depressive features such as anticipatory anhedonia and hypersomnia. They also had higher levels of psychomotor activity, but less anxiety.

\section{Bipolar depression and outcome}

In reviewing predictors of psychosocial outcome in bipolar disorder, MacQueen et al (2001) pooled data from 17 patient cohorts including a total of 1450 patients followed-up for variable periods of time, the longest being 15 years. Estimates of psychosocial impairment varied between $30 \%$ and $60 \%$. The number of past depressions (admissions or episodes) was a much stronger predictor of psychosocial outcome than the number of previous manic presentations. Bipolar disorder is also associated with cognitive dysfunction. The relationship between poor cognitive function and outcome has recently been examined in bipolar disorder, where it was found to predict occupational status (Dickerson et al, 2004). Number of depressive episodes, as well as the presence of even low-level, residual depressive symptoms, have been shown to have a significant negative impact on cognition in bipolar disorder patients (reviewed by Quraishi \& Frangou, 2002).

A significant complication in the treatment and outcome of bipolar disorder is the presence of comorbid psychiatric disorders. In the National Comorbidity Survey, $92.9 \%$ of the people with bipolar I disorder also met criteria for a lifetime anxiety disorder (Kessler et al, 1994, 1997). The Epidemiologic Catchment Area Study found 60.7\% lifetime prevalence for substance misuse or dependence in individuals with bipolar disorder (Regier et al, 1990). With regards to alcohol misuse or dependence, patients with bipolar disorder had much higher rates of comorbidity (about $40 \%$ ) than patients with other chronic mental disorders, including schizophrenia and unipolar depression. The impact of comorbidity on the treatment and outcome of bipolar depression has not been fully investigated, but clinicians should always consider this possibility, particularly in treatment-resistant patients. 
Finally, bipolar depression is associated with significant mortality. Between $10 \%$ and $15 \%$ of people with bipolar disorder complete suicide (DSM-IV, 1994) and about 40\% attempt it (Leverich et al, 2003). Although there are multiple factors associated with suicidality in bipolar disorder (including comorbidity with other Axis I, II and III diagnoses, family history of affective disorders, and suicide and symptom severity and chronicity) number of depressive episodes and/or hospitalisations remains an important predictor (Leverich et al, 2003). A population-based study identified 31 people who had had bipolar I disorder in an annual cohort of 1397 suicides. Most suicides occurred during a major depressive episode (79\%) and less commonly during mixed episodes $(11 \%)$ or psychotic mania $(11 \%)$ (Isometsa et al, 1994).

\section{Pharmacological approaches to the treatment of bipolar depression}

'Mood stabilisers' are considered the mainstay of treatment in bipolar disorder and these include lithium and anticonvulsants such valproate, carbamazepine and lamotrigine. The concept of mood stabiliser is currently being reviewed. In the past, demonstration of acute antidepressant and antimanic properties was considered sufficient, whereas today many authorities suggest that 'true' mood stabilisers should also prevent or reduce the risk of recurrence of mood episodes of either polarity (Bauer \& Mitchner, 2004). In this review, I use 'mood stabiliser' to denote lithium, valproate, carbamazepine or lamotrigine. Valproate (valproic acid) is usually administered as its sodium salt, but it is also available as valpromide (the amide of valproic acid) and divalproex sodium (a combination of sodium valproate and valproic acid in a 1:1 molar ratio). Here , the term valproate covers all forms unless otherwise specified. Lamotrigine was recently licensed for the treatment of bipolar disorder depression in Europe and Ireland, but not in the UK, where additional evidence was required. Below we discuss the pharmacotherapy of bipolar depression in terms of acute and long-term treatment, as defined in Box 2.

\section{Box 2 Definition of acute and long-term treatment}

- Acute treatment: focuses on symptomatic improvement of patients during an acute mood episode

- Long-term treatment: focuses on prevention of relapse or recurrence of mood episodes

\section{Treatment of acute bipolar depression}

\section{Lithium}

Several double-blind randomised controlled clinical trials (RCTs) from the 1970s confirmed the acute antidepressant effect of lithium compared with placebo, with response rates ranging from 64\% to $100 \%$ (Stokes et al, 1971; Noyes et al, 1974; Baron et al, 1975). However, the relative efficacy of lithium versus antidepressants in the treatment of bipolar depression remains unclear. One double-blind RCT found a higher level of symptom improvement with imipramine (58\%) than with lithium (32\%) (Fieve et al, 1968), whereas another found comparable improvements with the two treatments (Worrall et al, 1979). However, both studies were based on small samples $(n=29)$. Nemeroff et al (2001) approached this issue by comparing the efficacy of imipramine, paroxetine or placebo as adjuncts to lithium in the treatment of bipolar depression. Patients with high serum lithium levels did not derive any benefit from the addition of an antidepressant. In contrast, antidepressant response was significantly higher than placebo for both paroxetine and imipramine in patients with low serum lithium levels.

\section{Anticonvulsants}

The role of valproate in the acute treatment of bipolar depression is unclear. In an open prospective study, patients with rapid-cycling bipolar disorder $(n=78)$ were assigned according to prior treatment response to valproate monotherapy or as an add-on to their existing medication. A significant acute valproate treatment response was observed in $19 \%$ of patients with depressive and $87 \%$ of patients with mixed states (Calabrese et al, 1992). In a naturalistic study (Ghaemi \& Goodwin, 2001) based on reviews of medical records of patients presenting primarily with bipolar depression $(n=38)$, a significant antidepressant effect was seen for valproate, although it was used as monotherapy in only $24 \%$ of patients. In this study the efficacy of valproate was similar to that of lithium. However, in a more recent and rigorous 8-week study where patients with bipolar depression $(n=43)$ were randomised to valproate or placebo, no significant differences in efficacy were observed (Gyulai et al, 2003).

Response rates to carbamazepine in double-blind placebo-controlled trials of bipolar depression were found to be quite high (about 70\%), but this should be considered in the context of the very small sample sizes in these studies (total of 40 patients) (Ballenger \& Post, 1980; Post et al, 1983; Post et al, 1986). Also, the multiple interactions of carbamazepine with 
other medications make its use quite cumbersome (reviewed by Patsalos \& Perucca, 2003)

Calabrese et al (1999b) compared the efficacy of 50 and $200 \mathrm{mg} /$ day of lamotrigine monotherapy against placebo in a 7-week trial of 195 patients with bipolar I disorder depression. Improvement (defined as $50 \%$ reduction in scores on the MontgomeryÅsberg Depression Rating Scale) was noted in 54\% of patients in the $200 \mathrm{mg} /$ day group, $48 \%$ in the $50 \mathrm{mg}$ /day group and $29 \%$ in the placebo group; lamotrigine at $200 \mathrm{mg}$ / day showed clearer differentiation from placebo as early as week three.

A 48-week, open-label, prospective trial was conducted in 75 patients with bipolar I or II disorder who were intolerant or partially responsive to prior treatment and who presented in depressed, hypomanic, manic or mixed states (Calabrese et al, 1999a). Lamotrigine was used as an adjunct in 60 patients and as monotherapy in 15. Compared with baseline, depressed patients exhibited a $42 \%$ decrease in the Hamilton Rating Scale for Depression scores at the end of the study, and patients presenting with hypomania, mania or a mixed state exhibited a $74 \%$ decrease in Mania Rating Scale scores.

\section{Antidepressants}

The role of antidepressants in the treatment of bipolar depression remains unclear and largely controversial. Key uncertainties centre on response rates to antidepressants in bipolar disorder depression, antidepressant-induced polarity switch and optimal duration of antidepressant treatment.

Reported response rates in bipolar depression to a variety of antidepressants show significant variation. Imipramine has been the most studied tricyclic antidepressant (TCA) in bipolar disorder. A number of double-blind studies suggested that, on average, response rates to imipramine (and, by inference, to other TCAs) were in the region of 55\% (Baumhackl et al, 1989; Cohn et al, 1989; Himmelhoch et al, 1991; Thase et al, 1992). Treatment response to selective serotonin reuptake inhibitors (SSRIs) may be possibly marginally higher (Cohn et al, 1989; Simpson \& DePaulo, 1991). Other studies suggested that monoamine oxidase inhibitors (MAOIs) such as tranylcypromine and moclobemide may offer additional benefits for bipolar disorder patients with 'atypical' depressive features compared with TCAs (Baumhackl et al, 1989; Himmelhoch et al, 1991; Thase et al, 1992). Finally, a small double-blind study found similar efficacy for bupropion and desipramine in bipolar depression (Sachs et al, 1994). Post et al (2001a) focused on evaluating the augmenting effect of antidepressants in patients with bipolar disorder who were experiencing a major depressive episode despite adequate mood-stabilising treatment. In a double-blind design 64 patients were randomised to bupropion, sertraline or venlafaxine in the 10-week acute phase of the trial. Response rates were in the region of $37 \%$.

With regard to manic/hypomanic switches, a systematic review by Peet (1994) found these to occur significantly more often with TCAs $(11.2 \%)$ than with SSRIs $(3.7 \%)$ or placebo $(4.2 \%)$. Therefore, the recommended practice is to avoid TCAs and to prescribe antidepressants together with a mood stabiliser (i.e. lithium or anticonvulsants). However, several recent studies reported higher than expected switch rates. In the study by Post et al (2001a) a 14\% switch rate was observed during 10-week acute treatment with bupropion, sertraline or venlafaxine despite concomitant mood stabiliser treatment. Even higher rates were noted by Ghaemi et al (2004) in a retrospective observational study. Here the outcome of antidepressant treatment (based on medical records) of 41 patients with bipolar depression was compared with that of 37 patients with unipolar depression. The overall response rate to antidepressants was much lower in bipolar (about 50\%) than in unipolar depression (about 70\%). Mood stabilisers did not prevent cycle acceleration and rapid cycling, which occurred in $25.6 \%$ and $32.1 \%$ of patients respectively. Despite concomitant moodstabilising medication $31.6 \%$ of patients switched polarity; this occurred far more frequently (84.2\%) in patients not on mood stabilisers.

It is not yet clear what should be the optimal duration of antidepressant treatment in bipolar disorder depression. Data emerging from the Stanley Foundation Bipolar Network (Post et al, 2003), an international multicentre study, suggest that, for patients who respond well to antidepressants over a 2-month period, long-term treatment may be helpful in reducing further depressive relapses.

The uncertainty over the cost:benefit ratio of antidepressant use has led to the recommendation that bipolar depression be treated by optimising the dose of mood stabilisers or adding a second mood stabiliser if patients are already on an adequate dose of one. Young et al (2000) conducted a small RCT to evaluate the usefulness of the latter option. Twenty-seven patients with bipolar I disorder $(n=11)$ or bipolar II disorder $(n=16)$ experiencing a major depressive episode while on adequately dosed treatment of lithium or valproate were randomised to receive a second mood stabiliser (lithium or valproate) or paroxetine administered in a double-blind fashion for 6 weeks. Both interventions were equally effective in reducing the level of depression and improving patients' function. However, patients who received a second mood stabiliser were more likely to discontinue treatment, implying that the addition of an antidepressant was overall a better treatment option. 


\section{Antipsychotics}

The possibility that atypical antipsychotics (clozapine, risperidone, quetiapine and olanzapine) are useful in bipolar depression has been raised following reports of their antidepressant action in the treatment of psychotic disorders such as schizophrenia and schizoaffective disorder (Collaborative Working Group on Clinical Trial Evaluations, 1998). However, only olanzapine has been tested in placebo-controlled RCTs in bipolar depression.

Tohen et al (2003) examined the use of olanzapine and olanzapine-fluoxetine combination in the treatment of bipolar I disorder in an 8-week, double-blind RCT. Patients were randomised to placebo $(n=377)$; olanzapine ( $n=370 ; 5-20 \mathrm{mg} /$ day); or olanzapinefluoxetine combination $(n=86 ; 6$ and 25,6 and 50, or 12 and $50 \mathrm{mg}$ /day). Both active treatment groups showed statistically significant improvement in depressive symptoms compared with the placebo group. Greater improvement was noted in the olanzapine-fluoxetine group (response rate nearly $50 \%$ ) compared with the olanzapine group from week four onwards. The rate of manic switches was low (placebo, 6.7\%; olanzapine, 5.7\%; and olanzapinefluoxetine, $6.4 \%$ ) and did not differ significantly between the groups.

Vieta et al (2001) conducted a 6-month open-label study of adjunctive risperidone in 541 patients with bipolar or schizoaffective disorder presenting with manic, mixed or depressive episodes. The addition of risperidone led to significant reductions in manic and depressive symptoms rating as well as overall clinical improvement at week six. During the first 6 weeks of treatment, a very low incidence of exacerbation of mania was observed ( $2 \%)$.

In a retrospective chart review of 16 patients with bipolar disorder partially responding to adequately dosed lithium or valproate, the addition of quetiapine resulted in $56 \%$ being judged as much or very much improved in ratings of mania, depression and overall illness severity (Sokolski \& Denson, 2003).

\section{Long-term treatment}

\section{Lithium and anticonvulsants}

In the 1970s, Prien and colleagues published two studies on the long-term efficacy of lithium in bipolar disorder (Prien et al, 1973, 1974) that showed significant benefits in prophylaxis of both mania and depression. A recent meta-analysis of RCTs of lithium in bipolar disorder confirmed its efficacy in preventing manic episodes, but concluded that its protective effect against depressive recurrences was equivocal (Geddes et al, 2004). The role of lithium in the long-term treatment of bipolar disorder has been questioned primarily because its 'real-world' effectiveness was found to be much lower than its efficacy in clinical trials (Harrow et al, 1990). Patients in ordinary clinical care tend to discontinue longterm lithium treatment for a number of reasons, including side-effects, low acceptability of regular blood testing and less structured follow-up arrangements than in research settings. However, lithium may have particular efficacy in reducing suicidality in bipolar disorder. Most of the evidence for this comes from naturalistic studies comparing patient cohorts on and off lithium, but the findings are consistent and the reported anti-suicidal effect is large (Baldessarini et al, 2003). Existing evidence suggests that the anti-suicidal effects of lithium are probably superior to those of carbamazepine and valproate (Thies-Flechtner et al, 1996; Goodwin et al, 2003).

Only one placebo-controlled RCT has assessed the efficacy of carbamazepine in long-term treatment of bipolar disorder. This found that three times as many patients remained stable on carbamazepine over a period of 1 year than on placebo, but there were only 12 and 10 patients in the respective treatment arms (Okuma et al, 1981). Of the studies that used an active comparator, only one focused specifically on bipolar disorder (Greil et al, 1997). Patients with bipolar disorder $(n=144)$ were equally randomised to treatment with lithium or carbamazepine and followed-up for an average of 2.5 years. Lithium was found to be superior to carbamazepine, although no information regarding differential efficacy for depressive and manic recurrences was given.

Bowden et al (2000) conducted the only randomised placebo-controlled trial of valproate in bipolar disorder. Patients $(n=370)$ recovering from a manic episode were randomised on a 2:1:1 ratio to valproate, lithium or placebo and were followed up for 52 weeks. In the primary outcome measure, time to recurrence of any mood episode, no difference was found between placebo and valproate. Valproate was superior to placebo in terms of discontinuation rates because of a depressive episode and superior to lithium in terms of reducing deterioration of depressive symptoms. A much older study (Lambert \& Venaud, 1992) found lithium to have comparable efficacy to valpromide in reducing the number of episodes over a 2-year period in a sample of 150 patients (121 of whom with bipolar disorder and 29 with unipolar depression). However, no information regarding differential efficacy for preventing depressive or manic episodes was given.

Calabrese et al (2003) randomly assigned patients recovering from a depressive episode in bipolar disorder to lamotrigine $(n=221)$, lithium $(n=121)$ or placebo $(n=121)$ monotherapy and followed them up for 18 months. The primary outcome measure was time to intervention (i.e. addition of 
another medication) for an episode of any polarity. Time to intervention for any mood episode was statistically longer for both active treatments (median survival times for lamotrigine and lithium were 200 and 170 days respectively) compared with placebo (median survival time was 93 days). Lamotrigine was statistically superior to placebo at prolonging time to intervention for depression, and lithium was statistically more effective in prolonging time to intervention for mania. A similar pattern was observed for patients with bipolar I disorder $(n=175)$ randomised to lamotrigine $(n=59)$, lithium $(n=46)$ or placebo $(n=70)$ after an index manic episode and follow-up for 18 months (Bowden et al, 2003).

\section{Antidepressants}

Post et al (2001a) reported on 1-year blinded followup data for 42 patients with bipolar depression who had achieved remission of their index episode following addition of sertraline, venlafaxine or bupropion to their mood stabilisers. They found a switch rate into mania or hypomania of $33 \%$. However, the high risk of polarity switch has to be balanced against the considerable risk of relapse into depression following antidepressant withdrawal: at least one study found this to be nearly five times greater in bipolar disorder depression than unipolar depression (Ghaemi et al, 2004). This issue has been further highlighted by Altshuler et al (2003), who proposed that the risk of depressive relapse is significantly higher than that of a manic switch and that early discontinuation of antidepressant treatment may not always be advisable. In their study, 84 patients with bipolar disorder in remission after a depressive episode had been treated with an antidepressant added to their ongoing mood stabilisers were followed up for a period of 1 year. They report that 43 patients discontinued their antidepressant medication within 6 months of remission, whereas 41 continued antidepressant treatment. Seventy per cent of the patients who discontinued had a depressive relapse, as opposed to $36 \%$ of those who continued antidepressant treatment. The former also relapsed into depression earlier and had shorter periods of euthymia. Manic switches were observed in $15 \%$ of the sample, with a roughly equal split between the two groups of patients.

\section{Antipsychotics}

The significant reductions in manic and depressive symptoms and the overall clinical improvement at week six of Vieta et al's study of adjunctive risperidone in acute treatment mentioned above were maintained at month six (Vieta et al, 2001).
Altamura et al (2003) compared the efficacy of variable doses of quetiapine with that of mood stabilisers in 28 patients with bipolar disorder over a 12-month period. Patients were randomised to receive open-label treatment with either quetiapine or classic mood stabilisers. No significant differences were noted between the two treatment groups and all patients showed improvements in ratings of depression and overall illness severity.

Tohen et al (2003) conducted a 47-week randomised double-blind comparison of olanzapine and valproate in 251 patients with bipolar disorder who entered the study while in a manic or mixed episode. No differences were observed between the two treatments in subsequent relapse into mania or depression.

Clozapine may also have a role in treatmentresistant mood disorders, although the evidence is mostly based on case series and naturalistic studies. Patients in psychotic and manic/excitable states may derive most benefit from clozapine treatment (Zarate et al, 1995; Ciapparelli et al, 2003).

\section{Future trends}

Until fairly recently, the emphasis on mania when treating bipolar disorder overshadowed the need to develop our understanding of bipolar depression. Consequently, our evidence base is inadequate and there are many areas of uncertainty that limit the quality of treatment guidelines and recommendations. Atypical antipsychotics now have an established role in the treatment of acute mania, and their potential benefits in bipolar disorder depression for both acute and long-term treatment are being investigated quite intensively. Although initial results are promising, more data and direct clinical experience are needed before their role in the treatment of bipolar disorder becomes clearer. New research evidence is emerging from ongoing large studies supported by the Stanley Foundation (Post et al, 2001b) and the National Institute of Mental Health (the Systematic Treatment Enhancement Program for Bipolar Disorder; http://www. stepbd.org/research). The latter, abbreviated to STEP-BD, is a modular study that plans to enrol about 5000 patients with bipolar disorder from 20 centres. Key specific modules are designed as randomised clinical trials to determine the most effective treatments for bipolar disorder depression and to optimise treatment strategies for relapse prevention. Additional objectives are the validation of current subtypes of bipolar disorder in terms of clinical features and course and their relation to common comorbid conditions. Furthermore, STEP$\mathrm{BD}$ aims to examine and evaluate treatment options for patients presenting with acute mania, rapid 
cycling bipolar disorder or comorbid substance misuse.

This intense research interest and the increasing availability of new data hold the promise of improved clinical decision-making and outcomes in bipolar disorder.

\section{References}

Akiskal, H. S. \& Benazzi, F. (2004) Validating Kraepelin's two types of depressive mixed states: "depression with flight of ideas" and "excited depression". World Journal of Biological Psychiatry, 5, 107-113.

Akiskal, H. S., Coryell, W., Leon, A. C., et al (1994) The time course of nonchronic major depressive disorder. Uniformity across episodes and samples. National Institute of Mental Health Collaborative Program on the Psychobiology of Depression - Clinical Studies. Archives of General Psychiatry, 51, 405-410.

Altamura, A. C., Salvadori, D., Madaro D., et al (2003) Efficacy and tolerability of quetiapine in the treatment of bipolar disorder: preliminary evidence from a 12-month open-label study. Journal of Affective Disorders, 76, 267-271.

Altshuler, L., Suppes, T., Black, D., et al (2003) Impact of antidepressant discontinuation after acute bipolar depression remission on rates of depressive relapse at 1-year follow-up. American Journal of Psychiatry, 160, 1252-1262.

American Psychiatric Association (1994) Diagnostic and Statistical Manual of Mental Disorders (DSM-IV) (4th edn). Washington, DC: APA.

American Psychiatric Association (2002) Practice guideline for the treatment of patients with bipolar disorder (revision). American Journal of Psychiatry, 159 (suppl. 4), 1-50.

Angst, J., Gamma, A., Benazzi, F., et al (2003) Diagnostic issues in bipolar disorder. European Neuropsychopharmacology, 13 (suppl. 2), S43-50.

Baldessarini, R. J., Tondo, L. \& Hennen, J. (2003) Lithium treatment and suicide risk in major affective disorders: update and new findings. Journal of Clinical Psychiatry, 64 (suppl. 5), 44-52.

Ballenger, J. C. \& Post, R. M. (1980) Carbamazepine in manicdepressive illness: a new treatment. American Journal of Psychiatry, 137, 782-790.

Baron, M., Gershon, E. S., Rudy, V., et al (1975) Lithium carbonate response in depression. Prediction by unipolar/ bipolar illness, average-evoked response, catechol-O-methyl transferase, and family history. Archives of General Psychiatry, 32, 1107-1111.

Bauer, M. S. \& Mitchner, L. (2004) What is a "mood stabilizer"? An evidence-based response. American Journal of Psychiatry, 161, 3-18.

Baumhackl, U., Bizière K., Fischbach R., et al (1989) Efficacy and tolerability of moclobemide compared with imipramine in depressive disorder (DSM-III): an Austrian double-blind, multicentre study. British Journal of Psychiatry, 155 (suppl. 6), 785-835.

Bowden, C. L., Calabrese, J. R., McElroy, S. L., et al (2000) A randomized, placebo-controlled 12 -month trial of valproate and lithium in treatment of outpatients with bipolar I disorder. Valproate Maintenance Study Group. Archives of General Psychiatry, 57, 481-489.

Bowden, C. L., Calabrese, J. R., Sachs, G., et al (2003) Lamictal 606 Study Group. A placebo-controlled 18-month trial of lamotrigine and lithium maintenance treatment in recently manic or hypomanic patients with bipolar I disorder. Archives of General Psychiatry, 60, 392-400.

Calabrese, J. R., Markovitz, P. J., Kimmel, S. E., et al (1992) Spectrum of efficacy of valproate in 78 rapid-cycling bipolar patients. Journal of Clinical Psychopharmacology, 12 (suppl. 1), 535-565.

Calabrese, J. R., Bowden, C. L., McElroy, S. L., et al (1999a) Spectrum of activity of lamotrigine in treatment-refractory bipolar disorder. American Journal of Psychiatry, 156, 10191023.
Calabrese, J. R., Bowden, C. L., Sachs, G. S., et al (1999b) A double-blind placebo-controlled study of lamotrigine monotherapy in outpatients with bipolar I depression. Lamictal 602 Study Group. Journal of Clinical Psychiatry, 60, 79-88.

Calabrese, J. R., Bowden, C. L., Sachs, G. S., et al (2003) Lamictal 605 Study Group. A placebo-controlled 18-month trial of lamotrigine and lithium maintenance treatment in recently depressed patients with bipolar I disorder. Journal of Clinical Psychiatry, 64, 1013-1024.

Ciapparelli, A., Dell'Osso, L., Bandettini di Poggio, A., et al (2003) Clozapine in treatment-resistant patients with schizophrenia, schizoaffective disorder, or psychotic bipolar disorder: a naturalistic 48-month follow-up study. Journal of Clinical Psychiatry, 64, 451-458.

Cohn, J. B., Collins, G., Ashbrook E., et al (1989) A comparison of fluoxetine, imipramine and placebo in patients with bipolar depressive disorder. International Clinical Psychopharmacology, 4, 313-322.

Collaborative Working Group on Clinical Trial Evaluations (1998) Atypical antipsychotics for treatment of depression in schizophrenia and affective disorders. Journal of Clinical Psychiatry, 59 (suppl. 12), 41-45.

Dickerson, F. B., Boronow, J. J., Stallings, C. R., et al (2004) Association between cognitive functioning and employment status of persons with bipolar disorder. Psychiatric Services (Washington, D.C.), 55, 54-58.

Fieve, R. R., Platman, S. R. \& Plutchik, R. R. (1968) The use of lithium in affective disorders. I: Acute endogenous depression. American Journal of Psychiatry, 125, 487-491.

Geddes, J. R., Burgess, S., Hawton, K., et al (2004) Long-term lithium therapy for bipolar disorder: systematic review and meta-analysis of randomized controlled trials. American Journal of Psychiatry, 16, 217-222.

Ghaemi, S. N. \& Goodwin, F. K. (2001) Long-term naturalistic treatment of depressive symptoms in bipolar illness with valproate vs. lithium in the setting of minimal antidepressant use. Journal of Affective Disorders, 65, 281-287.

Ghaemi, S. N., Sachs, G. S., Chiou, A. M., et al (1999) Is bipolar disorder still underdiagnosed? Are antidepressants overutilized? Journal of Affective Disorders, 52, 135-144.

Ghaemi, S. N., Rosenquist, K. J., Ko, J. Y., et al (2004) Antidepressant treatment in bipolar versus unipolar depression. American Journal of Psychiatry, 161, 163-165.

Goodwin, G. M. (2003) Evidence-based guidelines for treating bipolar disorder: recommendations from the British Association for Psychopharmacology. Journal of Psychopharmacology, 17, 149-173.

Goodwin, F. K., Fireman, B., Simon, G. E., et al (2003) Suicide risk in bipolar disorder during treatment with lithium and divalproex. JAMA, 290, 1467-1473.

Greil, W., Ludwig-Mayerhofer, W., Erazo, N., et al (1997) Lithium versus carbamazepine in the maintenance treatment of bipolar disorders - a randomised study. Journal of Affective Disorders, 43, 151-161.

Gyulai, L., Bowden, C. L., McElroy, S. L., et al (2003) Maintenance efficacy of valproate in the prevention of bipolar depression. Neuropsychopharmacology, 28, 13741382.

Hantouche, E. G., Akiskal, H. S., Lancrenon, S., et al (1998) Systematic clinical methodology for validating bipolar-II disorder: data in mid-stream from a French national multisite study (EPIDEP). Journal of Affective Disorders, 50, 163173.

Harrow, M., Goldberg, J. F., Grossman, L. S., et al (1990) Outcome in manic disorders. A naturalistic follow-up study. Archives of General Psychiatry, 47, 665-671.

Himmelhoch, J. M., Thase, M. E., Mallinger, A. G., et al (1991) Tranylcypromine versus imipramine in anergic bipolar depression. American Journal of Psychiatry, 148, 910-916.

Isometsa, E. T., Henriksson, M. M., Aro, H. M., et al (1994) Suicide in bipolar disorder in Finland. American Journal of Psychiatry, 151, 1020-4102.

Judd, L. L., Akiskal, H. S., Schettler, P. J., et al (2002) The long-term natural history of the weekly symptomatic status of bipolar I disorder. Archives of General Psychiatry, 59, 530-537. 
Judd, L. L., Akiskal, H. S., Schettler, P. J., et al (2003) A prospective investigation of the natural history of the longterm weekly symptomatic status of bipolar II disorder. Archives of General Psychiatry, 60, 261-269.

Keller, M. B. (1988) The course of manic-depressive illness. Journal of Clinical Psychiatry, 49 (suppl.), 4-7.

Kessler, R. C., McGonagle, K. A., Zhao, S., et al (1994) Lifetime and 12-month prevalence of DSM-III-R psychiatric disorders in the United States: results from the National Comorbidity Survey. Archives of General Psychiatry, 51, 8-19.

Kessler, R. C., Rubinow, D. R., Holmes, C., et al (1997) The epidemiology of DSM-III-R bipolar I disorder in general population survey. Psychological Medicine, 27, 1079-1089.

Lambert, P. A. \& Venaud, G. (1992) Comparative study of valpromide versus lithium in treatment of affective disorders. Nervure: Journal de Psychiatrie, 5, 57-65.

Leverich, G. S., Altshuler, L. L., Frye, M. A., et al (2003) Factors associated with suicide attempts in 648 patients with bipolar disorder in the Stanley Foundation Bipolar Network. Journal of Clinical Psychiatry, 64, 506-15.

Lish, J. D., Dime-Meenan, S., Whybrow, P. C., et al (1994) The National Depressive and Manic-Depressive Association (DMDA) survey of bipolar members. Journal of Affective Disorders, 31, 281-294.

MacQueen, G. M., Glenda, M., Young, L., et al (2001) A review of psychosocial outcome in patients with bipolar disorder Acta Psychiatrica Scandinavica, 103, 163-170.

Mitchell, P. B., Wilhelm, K., Parker, G., et al (2001) The clinical features of bipolar depression: a comparison with matched major depressive disorder patients. Journal of Clinical Psychiatry, 62, 212-216.

Nemeroff, C. B., Evans, D. L., Gyulai, L., et al (2001) Doubleblind, placebo-controlled comparison of imipramine and paroxetine in the treatment of bipolar depression. American Journal of Psychiatry, 58, 906-912.

Noyes, R., Dempsey, G. M., Blum, A., et al (1974) Lithium treatment in depression. Comprehensive Psychiatry, 15, 187193.

Okuma, T., Inanaga, K., Otsuki, S., et al (1981) A preliminary double-blind study on the efficacy of carbamazepine in prophylaxis of manic-depressive illness. Psychopharmacology, 73, 95-96.

Patsalos, P. N. \& Perucca, E. (2003) Clinically important drug interactions in epilepsy: interactions between antiepileptic drugs and other drugs. Lancet Neurology, 2, 473-481.

Peet, M. (1994) Induction of mania with selective serotonin re-uptake inhibitors and tricyclic antidepressants. British Journal of Psychiatry, 164, 549-550.

Post, R. M., Uhde, T. W., Ballenger, J. C., et al (1983) Carbamazepine and its $-10,11$-epoxide metabolite in plasma and CSF: relationship to antidepressant response. Archives of General Psychiatry, 1983, 40, 673-676.

Post, R. M., Uhde, T. W., Roy-Byrne, P. P., et al (1986) Antidepressant effects of carbamazepine. The American Journal of Psychiatry, 143, 29-34.

Post, R. M., Altshuler, L. L., Frye, M. A., et al (2001a) Rate of switch in bipolar patients prospectively treated with secondgeneration antidepressants as augmentation to mood stabilizers. Bipolar Disorders, 3, 259-265.

Post, R. M., Nolen, W. A., Kupka, R. W, et al (2001b) The Stanley Foundation Bipolar Network. I. Rationale and methods. British Journal of Psychiatry, 178 (suppl. 41), s169s176.

Post, R. M., Leverich, G. S., Nolen, W. A., et al (2003) A reevaluation of the role of antidepressants in the treatment of bipolar depression: data from the Stanley Foundation Bipolar Network. Bipolar Disorders, 5, 396-406.

Prien, R. F., Caffey, E. M. Jr. \& Klett, C. J. (1973) Prophylactic efficacy of lithium carbonate in manic-depressive illness: report of the Veteran's Administration and National Institute of Mental Health collaborative study group. Archives of General Psychiatry, 28, 337-341.

Prien, R. F., Klett, C. J. \& Caffey, E. M. (1974) Lithium prophylaxis in recurrent affective illness. American Journal of Psychiatry, 131, 198-203.
Quraishi, S. \& Frangou, S. (2002) Neuropsychology of bipolar disorder: a review. Journal of Affective Disorder, 72, 209-226.

Raymont, V., Bettany, D. \& Frangou, S. (2003) The Maudsley bipolar disorder project. Clinical characteristics of bipolar disorder I in a Catchment area treatment sample. European Psychiatry, 18, 13-17.

Regier, D. A., Farmer, M. E. \& Rae, D. S. (1990) Comorbidity of mental disorders with alcohol and other drug abuse: results from the Epidemiologic Catchment Area (ECA) Study. JAMA 264, 2511-2518.

Sachs, G. S., Lafer, B., Stoll, A. L., et al (1994) A double-blind trial of bupropion versus desipramine for bipolar depression. Journal of Clinical Psychiatry, 55, 391-393.

Simpson, S. G. \& DePaulo, J. R. (1991) Fluoxetine treatment of bipolar II depression. Journal of Clinical Psychopharmacology, 11, 52-54.

Sokolski, K. N. \& Denson, T. F. (2003) Adjunctive quetiapine in bipolar patients partially responsive to lithium or valproate. Progress in Neuro-Psychopharmacology and Biological Psychiatry, $27,863-866$

Stokes, P. E., Shamoian, C. A., Stoll, P. M., et al (1971) Efficacy of lithium as acute treatment of manic-depressive illness. Lancet, 1, 319-325.

Thase, M. E., Mallinger, A. G., McKnight, D., et al (1992) Treatment of imipramine-resistant recurrent depression. IV A double-blind crossover study of tranylcypromine for anergic bipolar depression. American Journal of Psychiatry, 149, 195198.

Thies-Flechtner, K., Muller-Oerlinghausen, B., Seibert, W., et al (1996) Effect of prophylactic treatment on suicide risk in patients with major affective disorders. Data from a randomized prospective trial. Pharmacopsychiatry, 29, 103107.

Tohen, M., Vieta, E., Calabrese, J., et al (2003) Efficacy of olanzapine and olanzapine-fluoxetine combination in the treatment of bipolar I depression. Archives of General Psychiatry, 60, 1079-1088.

Vieta, E., Goikolea, J. M., Corbella, B., et al, (2001) Risperidone safety and efficacy in the treatment of bipolar and schizoaffective disorders: results from a 6-month, multicenter open study. Journal of Clinical Psychiatry, 62, 818-825.

World Health Organization (1992) The ICD-10 Classification of Mental and Behavioural Disorders: Clinical Descriptions and Diagnostic Guidelines. Geneva: World Health Organization.

Worrall, E. P., Moody, J. P., Peet, M., et al (1979) Controlled studies of the acute antidepressant effects of lithium. British Journal of Psychiatry, 135, 255-262.

Yatham, L. N., Srisurapanont, M., Zis, A. P., et al (1997) Comparative studies of the biological distinction between unipolar and bipolar depressions. Life Science, 61, 1445-1455.

Young, L. T., Joffe, R. T., Robb, J. C., et al (2000) Double-blind comparison of addition of a second mood stabilizer versus an antidepressant to an initial mood stabilizer for treatment of patients with bipolar depression. American Journal of Psychiatry, 157, 124-126.

Zarate, C. A. Jr., Tohen, M. \& Baldessarini, R. J. (1995) Clozapine in severe mood disorders. Journal of Clinical Psychiatry, 56, $411-417$.

\section{MCQs}

\section{Patients with bipolar disorder:}

a have more minor/subsyndromal depressive symptoms than depressive episodes

b are more likely to attempt suicide when depressed than when manic

c are symptomatic for up to $50 \%$ of the time

$\mathrm{d}$ are often misdiagnosed as having major depressive disorder

e are less likely than patients with major depressive disorder to experience psychosis during depressive episodes. 
2 Lithium:

a has acute antidepressant effects in patients with bipolar depression

b monotherapy has been consistently shown to lead to higher response rates than antidepressants in the treatment of bipolar depression

c is equally effective in preventing manic and depressive relapses

$\mathrm{d}$ is superior to carbamazepine in the long-term treatment of bipolar disorder

e may reduce suicide risk in patients with bipolar disorder.

\section{Antidepressants:}

a may be less effective in the treatment of major depression than bipolar depression

$\mathrm{b}$ are associated with higher rates of depressive relapse on discontinuation in patients with bipolar disorder than in those with major depression

c carry a significant risk of inducing cycle acceleration or manic switch in bipolar disorder

$\mathrm{d}$ should be used in combination with a mood stabiliser

e may have a role in preventing depressive relapse in the long-term treatment of bipolar disorder.
4 Randomised controlled trials have shown that:

a lamotrigine has acute antidepressant effects in bipolar depression

b valproate is superior to placebo in the treatment of acute bipolar depression

c long-term treatment with lamotrigine is equally effective in prolonging time to intervention for manic and depressive episodes

d valproate may be superior to lithium in preventing deterioration of depressive symptoms

e in acute bipolar depression, treatment with a combination of two mood stabilisers may be as effective in reducing depressive symptoms as the combination of a mood stabiliser and an antidepressant.

5 In the long-term treatment of bipolar disorder:

a monotherapy with a mood stabiliser is not indicated

b olanzapine may be more effective than valproate

c adjunctive treatment with atypical antipsychotics may improve symptom control

d antidepressants or lamotrigine can be considered in combination with mood stabilisers when depressive symptoms predominate

e clozapine may have a role in the treatment of schizoaffective but not bipolar disorder. 